\title{
RESISTENCIA A LA COMPRESIÓN AXIAL SIMPLE DE BLOQUES HUECOS DE CONCRETO ELABORADOS CON FIBRAS DE POLIPROPILENO
}

\author{
Compressive axial strenght of hollow concrete blocks fabricated with polypropylene fibers

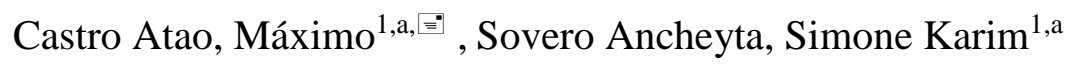 \\ ${ }^{1}$ Universidad Andina del Cusco, Cusco, Perú. \\ a Ingeniería civil \\ ”mcaing21@gmail.com
}

\begin{abstract}
Resumen
En el campo de la construcción es importante contar con materiales que cumplan las características adecuadas que se exigen en las normas para construir edificaciones seguras y resistentes. Los bloques huecos de concreto (BHC), que se fabrican en la ciudad del Cusco, no cumplen con estas características, porque se fabrican de manera artesanal y semindustrial. Por otro lado, es sabido que este tipo de unidades tienden a fisurarse (si no se sigue un proceso de curado adecuado). Muchas investigaciones se han llevado a cabo para demostrar que las fibras de polipropileno (Fpp), son un material adecuado para controlar los efectos de fisuración en elementos de concreto, pero no se han obtenido resultados contundentes con respecto a la modificación en su resistencia. Por este motivo, en esta investigación se han elaborado setenta y cinco BHC adicionados con Fpp, en dosificaciones de 300 g, 600 g, 750 g, y 900 g, por unidad, y se han ensayado por compresión axial simple a diferentes edades de: 7, 14, 21, 28 y 56 días. Como resultado, se ha observado que las Fpp incrementan la resistencia de los BHC hasta en un 90\%, lo que permite obtener bloques que pueden ser catalogados como bloques tipo $\mathrm{P}\left(\mathrm{f}^{\prime} \mathrm{b}>50 \mathrm{~kg} / \mathrm{cm}^{2}\right.$ ), considerados adecuados para ser estructurales en la construcción de edificaciones de mampostería.

Palabras clave: bloques huecos de concreto, fibras de polipropileno, resistencia característica a la compresión.
\end{abstract}

\begin{abstract}
In the field of construction, it is important to have materials that meet the appropriate characteristics that are required in the standards to build safe and resistant buildings. The hollow blocks of concrete (BHC), which are manufactured in the city of Cusco, do not meet these characteristics, because they are manufactured in an artisanal and semi-industrial manner. On the other hand, it is known that this type of units tends to crack if an adequate curing process is not followed. Many investigations have been carried out to demonstrate that polypropylene fibers (Fpp), are an adequate material to control the effects of cracking in concrete elements but, no conclusive results have been obtained regarding the modification in their resistance. For this reason, seventy-five BHC added with Fpp have been developed in this investigation, in dosages of $300 \mathrm{gr}, 600 \mathrm{gr}, 750 \mathrm{gr}$ and $900 \mathrm{gr}$ per unit, and have been tested by simple axial compression at different ages of:7, 14, 21, 28 and 56 days. As a result, it has been it has been observed that the Fpp increase the resistance of the BHC by up to $90 \%$, which allows obtaining blocks that can be classified as P-type blocks (f'b $\left.>50 \mathrm{~kg} / \mathrm{cm}^{2}\right), \mathrm{considered}$ suitable for being structural in the construction of masonry buildings.
\end{abstract}

Keywords: concrete hollow blocks, polypropylene fibers, characteristic resistance to compression.

Citar como: Castro, M., Sovero, S. (2018). Resistencia a la compresión axial simple de bloques huecos de concreto elaborados con fibras de polipropileno. Rev Yachay, 7(1),389-395.

Recibido: 16-09-2018; Aceptado 07-12-2018

\section{Introducción}

En la ciudad de Cusco se encuentran muy pocas edificaciones de albañilería estructural, debido a que no se cuenta con ladrillos o bloques que cumplan con los parámetros mínimos de resistencia que se especifican en la norma E.070 de albañilería (RNE 2006). Si bien en la ciudad existen muchas ladrilleras que fabrican unidades de arcilla cocida y bloqueteras que fabrican bloques huecos de concreto (BHC), estas unidades no cumplen con los parámetros mínimos de calidad para ser usadas como elementos estructurales en la construcción de

Rev. Yachay volumen (7) Número (1), enero-diciembre 2018 
muros portantes. Por este motivo, la mayoría de las edificaciones son de concreto armado, con un sistema de pórticos o dual, que consiste en un conjunto de pórticos - o estos rigidizados con muros de corte (placas)—, respectivamente. Esta situación, hace que los pobladores de la ciudad del Cusco, no cuenten con suficientes alternativas para elegir materiales de construcción que pudieran ser más económicos y que, a su vez, permitan definir espacios arquitectónicos más agradables para el usuario.

La deficiente calidad de las unidades de albañilería se debe principalmente, a que estas se producen en condiciones no estandarizadas y de manera manual, utilizando procedimientos muy precarios y sin ningún control de calidad (Gallegos, H., Casabonne, C., 2006). En el caso de las bloqueteras, la falta de control de calidad hace que la resistencia a la compresión de las unidades tipo BHC sea menor a $20 \mathrm{~kg} / \mathrm{cm}^{2}$, lo que significa que estas unidades no se pueden usar, ni siquiera como elementos para construir muros no estructurales de tabiquería.

Por lo tanto, se requiere de un método y materiales adecuados que ayuden a mejorar la calidad de los BHC. Estudios recientes han demostrado que el concreto se comporta de mejor manera cuando se le agregan fibras sintéticas. Estas fibras ayudan a reducir el proceso de fisuración y agrietamiento del concreto producto de los cambios volumétricos por la pérdida de agua en su proceso de curado.

Zainab Z. Ismail; Enas A. AL-Hashmi, (2008), remplazaron el agregado del concreto por restos de plástico, compuesto por $20 \%$ de poliestireno y $80 \%$ de polietileno, y obtuvieron concretos con una significativa reducción de las microfisuras.

Por otro lado, Bandodkar, L., Gaonkar, A., Gaonkar,N. \& Gauns, Y., (2011), remplazaron el 10\% de arena del agregado fino del concreto por restos pulverizados de botellas plásticas, obteniendo una reducción de hasta 13,5\% en la resistencia a la compresión del concreto.

De acuerdo con (Armas Aguilar, 2016), al usar fibras de polipropileno con una dosificación de $400 \mathrm{gr} / \mathrm{m}^{3}$ se logra reducir la fisuración del concreto hasta en un $90 \%$, y se incrementa la resistencia a la compresión y flexión del concreto entre el 3\% y el 14\%, respectivamente.

Al observar la mejora de las características mecánicas del concreto, en la presente investigación se propone mejorar la resistencia a la compresión de los BHC. En primer término, se ha planteado modificar la dosificación comercial de los componentes (cemento y agregados) que se usan en la fabricación de los BHC, con el fin de que alcancen una resistencia mínima de por lo menos $20 \mathrm{~kg} / \mathrm{cm}^{2}, \mathrm{y}$ puedan ser catalogadas como bloques tipo NP. Luego, a estos bloques mejorados se les adicionaron fibras de polipropileno (Fpp) en la mezcla de fabricación (manteniendo constantes los otros componentes) para medir el incremento de la resistencia a la compresión, la cual supera los $50 \mathrm{~kg} / \mathrm{cm}^{2}$, que los cataloga como bloques tipo P. Tanto la modificación en la dosificación de componentes, como la adición de fibras se realizó de forma paramétrica. En el caso de las Fpp, estas se adicionaron en proporciones de 0, 300, 600, 750, y 900 gramos por unidad.

\section{Objetivos}

El objetivo general es evaluar la resistencia a la compresión axial simple de bloques huecos de concreto elaborados con fibras de polipropileno y en diferentes proporciones.

Como objetivos específicos se han planteado:

- Evaluar cuál es la resistencia a la compresión de los bloques huecos de concreto que se fabrican en la ciudad de Cusco.

- Establecer una dosificación adecuada para que estos bloques huecos de concreto lleguen a clasificar, al menos, como bloques NP (no portantes), de acuerdo a la norma E.070 (2006).

- Medir cómo varía la resistencia a la compresión de los bloques huecos de concreto a medida que se varía la cantidad de fibras de polipropileno que se le añade a su fabricación.

\section{Materiales y métodos}

Diseño de la investigación

En la figura $n .^{\circ}$, se muestra el procedimiento que se siguió para llevar a cabo la presente investigación. Como punto de partida se identificaron las empresas que producen bloques huecos de concreto en el distrito de San Jerónimo, Cusco. Se eligió aquella cuyo proceso de fabricación es más estandarizado —en este caso, semindustrial一, y se ensayaron a compresión simple 6 especímenes. Debido a que la resistencia de los bloques fabricados en esta empresa era insuficiente para clasificarlos como NP (NTE E.070), se estudiaron las características y cantidades de los materiales que los conforman para determinar una nueva dosificación. Se plantearon 4 nuevas dosificaciones basadas en la variación de la cantidad de cemento y, se ensayaron 3 especímenes de cada una, con el fin de determinar la dosificación óptima para obtener un bloque tipo NP. Determinada esta dosificación óptima, se incorporaron las Fpp en cantidades de 300, 600, 750 y 900 gramos por cada unidad elaborada y se sometieron a ensayos de compresión simple a diferentes edades y así, verificar su 
resistencia. Para cada dosificación de Fpp, se ensayaron 15 especímenes (75 en total) a diferentes edades, 7, 14, 21, 28 y 56 días (tres por cada edad). Con los resultados obtenidos se ha evaluado si se cumplieron los objetivos propuestos.

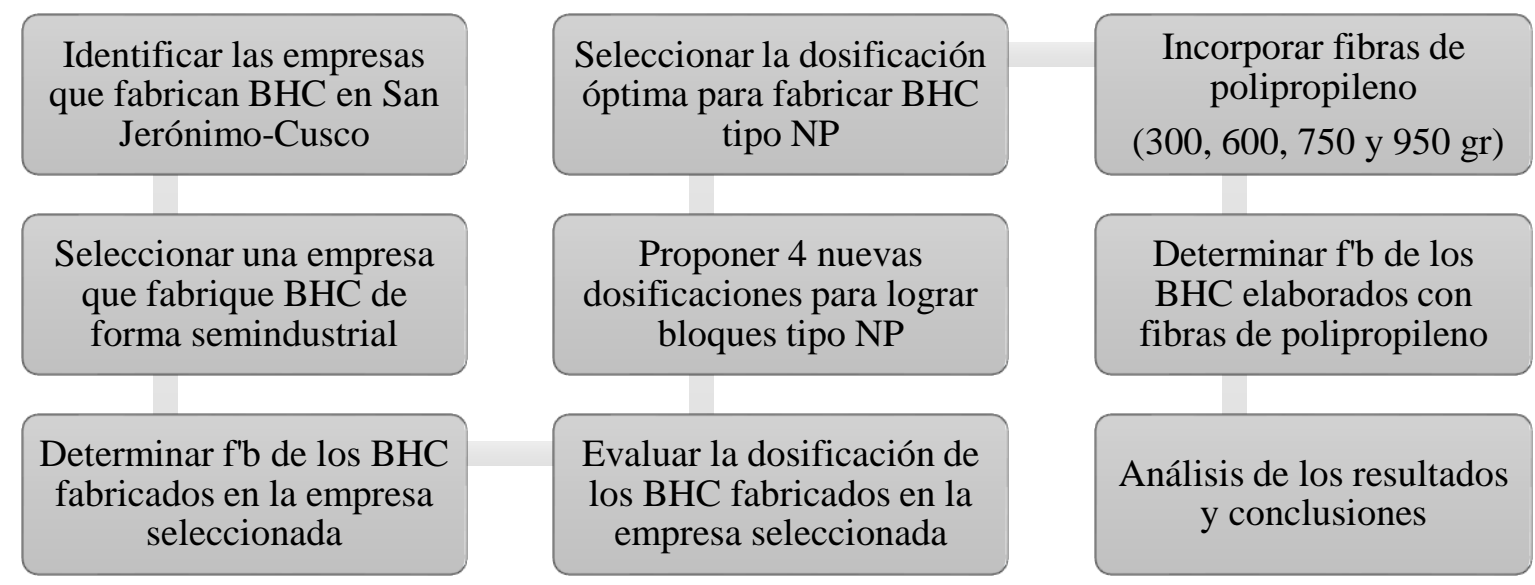

Figura n. ${ }^{\circ}$ : Diseño de la investigación

\section{Materiales y equipos}

Los materiales usados en la investigación fueron aquellos que se emplean de manera comercial en la ciudad de Cusco. En la tabla n. ${ }^{\circ} 1$, se muestran los materiales empleados en esta investigación y un resumen de sus características.

Tabla n. ${ }^{\circ}$ :

Materiales empleados en la fabricación de los BHC

\begin{tabular}{ll}
\hline \multicolumn{1}{c}{ Material } & \multicolumn{1}{c}{ Características generales } \\
\hline Cemento Portland puzolánico & Marca 'Yura' (Tipo IP) \\
Piedra chancada & Cantera “La Rinconada 2" (P’iñipampa) \\
Confitillo & Cantera de Zurite \\
Arena gruesa & Cantera de Huambutío \\
Agua & Potable \\
Fibras de polipropileno (Fpp) & Marca Z-aditivos (Lmax =35 mm) \\
\hline
\end{tabular}

La fabricación de los BHC, se realizó con los equipos de la empresa seleccionada. El equipo más relevante es la vibro compactadora, que permite fabricar los bloques de manera semindustrial (figura 2a) y, por otro lado, los moldes metálicos (figura 2b), que permite que las dimensiones de los BHC se estandaricen. Los ensayos se realizaron en la Universidad Andina del Cusco, y el principal equipo empleado fue la máquina de ensayo a compresión axial marca ACCU-TEK, con una capacidad de carga con resistencia de $1112 \mathrm{kN}(113,40 \mathrm{t}$, aproximadamente (figura 2c). 


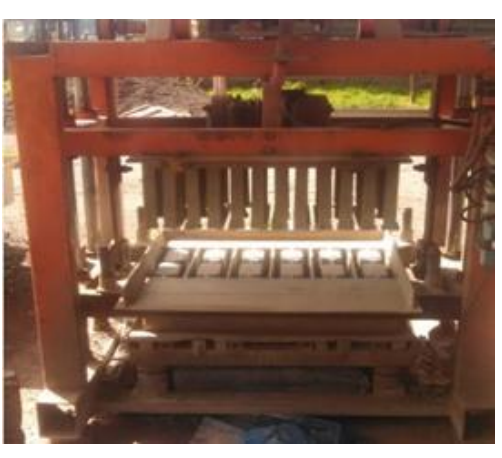

(a)

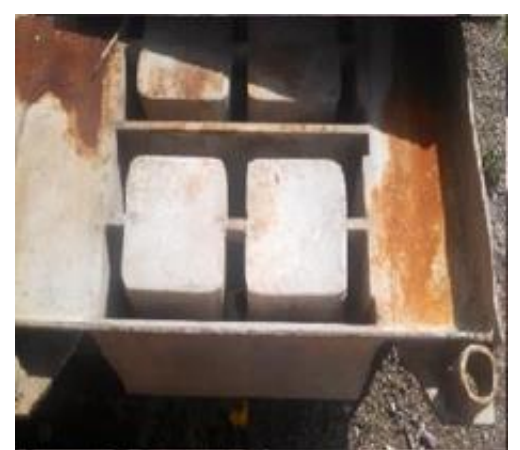

(b)

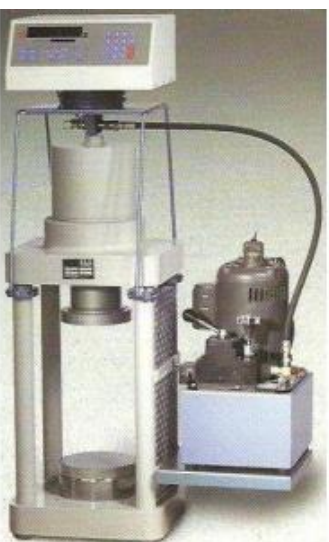

(c)

Figura.$^{\circ}$ 2: Principales equipos usados, a) vibrocompactadora, b) moldes metálicos para fabricar BHC y, c) máquina de compresión axial.

\section{Procedimiento}

La empresa seleccionada fabrica menos de 10000 unidades mensuales, por lo que, el muestreo inicial fue de 6 unidades (NTP 399.604, 2002). Estas unidades se fabricaron siguiendo el proceso común de la empresa seleccionada y luego se sometieron a pruebas de compresión simple para evaluar su resistencia. En seguida, se realizó un análisis de los materiales que se empleaban en la empresa y se planteó una dosificación más apropiada para obtener bloques tipo NP (f'b>20 kg/ $\left.\mathrm{cm}^{2}\right)$. Con la dosificación mejorada, se fabricaron 75 bloques para ensayarlos a compresión simple: a la edad de 7, 14, 21, 28 y 56 días, con dosificaciones de 0, 300 g, 600 g, 750 g y 900 g, de Fpp por unidad, tal como se indica en la tabla n. ${ }^{\circ} 2$.

Las dimensiones de los BHC fueron de 15 x 20 x $40 \mathrm{~cm}$, con dos alveolos por cada unidad y el espesor mínimo de las paredes fue de 2,5 $\mathrm{cm}$. Las fibras se añadieron como insumo final a la mezcla (figura 3a), cuyo batido se realizó en un tambor cilíndrico electrónico. Al desencofrar los BHC, fue posible observar las fibras en las superficies, de manera uniformemente distribuida, tal como se ve en la figura $3 b$.

Tabla n. ${ }^{\circ}$ 2: Cantidad de especímenes elaborados

\begin{tabular}{cccccc}
\hline \multirow{2}{*}{ Cantidad de Fpp (g/und) } & \multicolumn{5}{c}{ Tiempo de curado (días) } \\
\cline { 2 - 5 } & 7 & 14 & 21 & 28 & 56 \\
\hline 0 & 3 & 3 & 3 & 3 & 3 \\
300 & 3 & 3 & 3 & 3 & 3 \\
600 & 3 & 3 & 3 & 3 & 3 \\
750 & 3 & 3 & 3 & 3 & 3 \\
900 & 3 & 3 & 3 & 3 & 3 \\
\hline Total & 15 & 15 & 15 & 15 & 15 \\
\hline
\end{tabular}

En los tres primeros días, el curado de los BHC fue por aspersión y los días posteriores fue cubrirlas con bolsas de cemento vacías y humedecidas, recubiertas por plástico, tal como se muestra en la figura 3c. (Armas Aguilar C. H., 2016). 


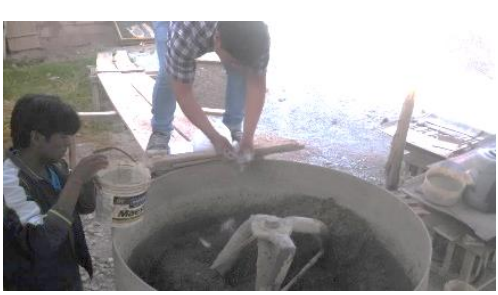

(a)

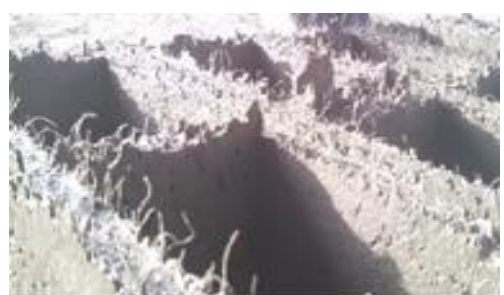

(b)

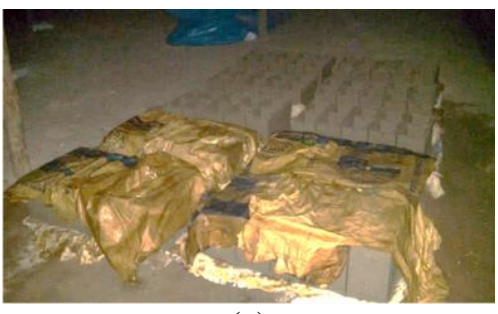

(c)

Figura n. ${ }^{\circ}$ 3: Elaboración de los BHC: a) batido de los materiales, b) Fpp visibles en la superficie y c) proceso de curado mediante bolsas de cemento

Para ensayar los bloques, estos se colocaron en la máquina de compresión axial en una posición tal que el centroide de las superficies de cada bloque quedó alineado con el centro de aplicación de la presión de soporte esférico de la máquina de ensayos (figuras 4a y 4b). Para uniformizar las superficies se usó un refrentado de yeso-cemento y, debido a que los discos de la máquina de ensayos no cubrían la superficie total de los especímenes, se colocaron dos planchas metálicas de 1" de espesor (ASTM C140-11a, 2014). La aplicación de la carga se hizo en dos etapas: primero, hasta la mitad de la carga esperada y segundo hasta la carga última.

En esta segunda etapa, se ajustaron los controles del equipo para que la cabeza móvil avance a una velocidad uniforme de tal manera, que la carga remanente se aplique en un tiempo no menor de 1 minuto ni mayor de 2 minutos. La falla de los BHC fue por aplastamiento, falla característica de este tipo de material (figura 4c).

Finalmente, la resistencia a la compresión característica de los bloques se obtiene al restar la desviación estándar del promedio de los tres ensayos realizados para cada caso (NTE E.070, 2016).

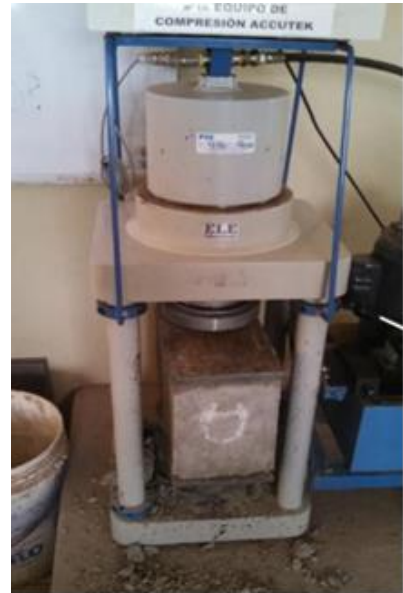

(a)

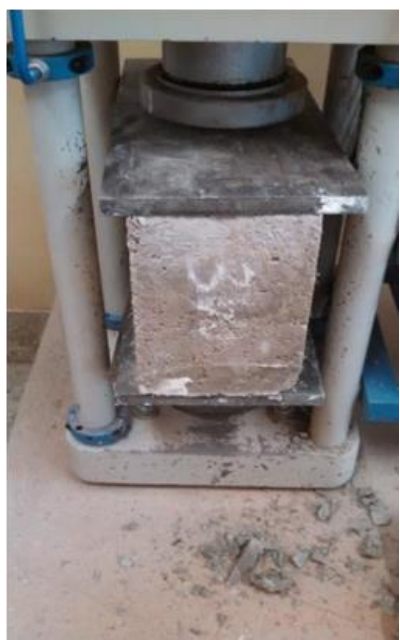

(b)

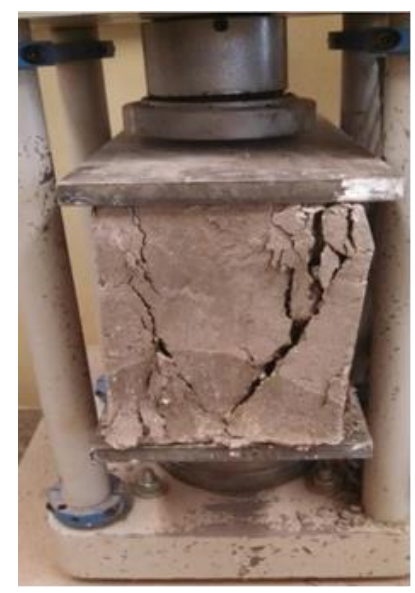

(c)

Figura 4: Ensayo a compresión: a) y b) colocación de los BHC en la máquina de ensayos, y c) falla característica de los $\mathrm{BHC}$ 


\section{Resultados}

En la tabla $n .^{\circ} 3$ se muestra la resistencia característica a compresión (f'b) de los BHC, en función de las diferentes dosificaciones de los materiales en porcentaje, sin la adición de Fpp.

\begin{tabular}{cccccc}
\multicolumn{2}{c}{ Tabla n. ${ }^{\circ}$ 3: Resistencia característica a compresión en función de la dosificación de materiales (sin Fpp) } \\
\cline { 2 - 4 } $\begin{array}{c}\text { Tipo de } \\
\text { Mezcla }\end{array}$ & Cemento & $\begin{array}{c}\text { Posificación de materiales }\left(\mathrm{m}^{3}\right) \\
\text { chanchada }\end{array}$ & Confitillo & $\begin{array}{c}\text { Arena } \\
\text { gruesa }\end{array}$ & $\begin{array}{c}\text { Resistencia característica a } \\
\text { compresión f'b (kg/cm2) }\end{array}$ \\
\hline Actual & 0,014 & 0,020 & 0,060 & 0,020 & 15,76 \\
Mezcla 1 & 0,016 & 0,019 & 0,059 & 0,019 & 17,94 \\
Mezcla 2 & 0,018 & 0,018 & 0,058 & 0,018 & 18,42 \\
Mezcla 3 & 0,021 & 0,017 & 0,057 & 0,017 & 20,47 \\
Mezcla 4 & 0,023 & 0,016 & 0,056 & 0,016 & 22,66 \\
\hline
\end{tabular}

En la figura $n .^{\circ} 5$, se exponen los resultados de la resistencia a la compresión axial obtenida en los bloques huecos de concreto para diferentes edades de ensayo y diferentes proporciones de Fpp.

En la tabla $n .^{\circ}$ 4, se revela el incremento de la resistencia característica a la compresión (f'b) de los BHC con Fpp, con respecto a los BHC sin Fpp

Tabla n. ${ }^{\circ}$ 4: Incremento de la resistencia a compresión de BHC sin Fpp y BHC con Fpp (28 días de edad).

\begin{tabular}{|c|c|c|}
\hline Dosificación con FPP (gr/und) & $\begin{array}{l}\text { Resistencia característica a compresión a los } 28 \text { días, } \\
\mathrm{f}^{\prime} \mathrm{b}\left(\mathrm{kg} / \mathrm{cm}^{2}\right)\end{array}$ & Incremento de resistencia (\%) \\
\hline 0 & 30,26 & \\
\hline 300 & 49,97 & 65,13 \\
\hline 600 & 55,21 & 82,45 \\
\hline 750 & 58,37 & 92,89 \\
\hline 900 & 52,39 & 73,13 \\
\hline
\end{tabular}

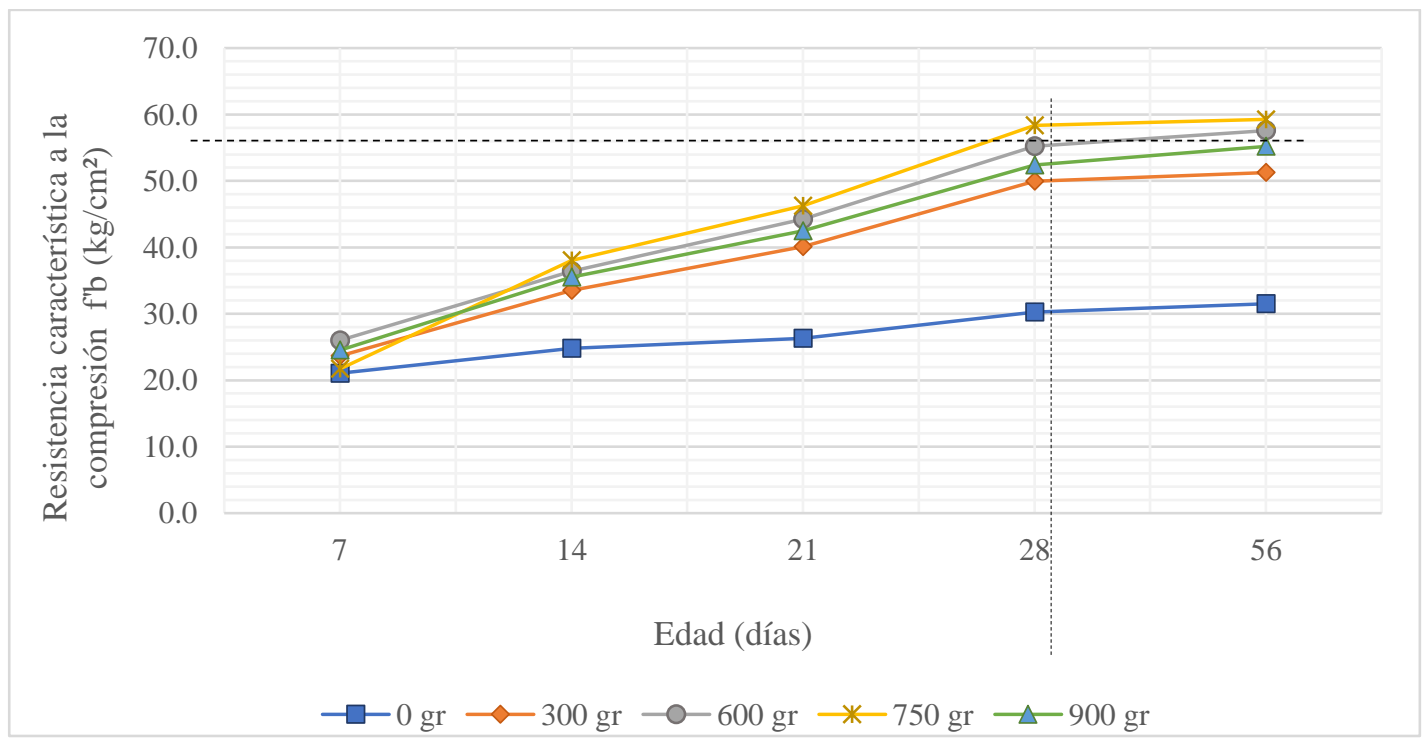

Figura . $^{\circ}$ 5: Resultados de los ensayos de compresión axial de los BHC a diferentes edades 


\section{Discusión}

En la tabla.$^{\circ}$ 3, se puede observar que se hizo necesario modificar la dosificación de los materiales para poder obtener resistencias a la compresión mayores o iguales a $20 \mathrm{~kg} / \mathrm{cm}^{2}$, de tal manera que los BHC fabricados en la empresa puedan catalogar como bloques tipo NP.

Para esta investigación se tomó como óptima la mezcla 4, cuya dosificación es de 0,023 $\mathrm{m}^{3}$ de cemento, $0,016 \mathrm{~m}^{3}$ de piedra chancada, $0,056 \mathrm{~m}^{3}$ de confitillo y $0,016 \mathrm{~m}^{3}$ de arena gruesa. A los BHC con esta dosificación se les añadió las Fpp en diferentes proporciones. En la figura.$^{\circ} 5$, se puede observar que a medida que se incrementa la cantidad de Fpp, la resistencia a la compresión se incrementa. La resistencia máxima de $59,27 \mathrm{~kg} / \mathrm{cm}^{2}$, se obtuvo a los 56 días de edad; al añadir $750 \mathrm{~g} / \mathrm{unidad}$ de Fpp. En la tabla n. ${ }^{\circ} 4$, se muestra el incremento de resistencia de los BHC adicionados con Fpp y ensayados a los 28 días (edad establecida por norma). En esta tabla, se puede apreciar que la resistencia a la compresión se incrementa desde que se le añade la mínima cantidad de Fpp (300 g/unidad) y que con esta dosificación ya se obtienen BHC que pueden clasificar como tipo P, con resistencias iguales o mayores a $50 \mathrm{~kg} / \mathrm{cm}^{2}$.

\section{Conclusiones}

Como conclusiones de la presente investigación, se pueden indicar las siguientes:

- En la ciudad de Cusco, existen muy pocas fábricas de BHC que elaboren unidades con las características adecuadas para ser usadas de manera estructural en la construcción. Esto es debido a la deficiencia en los procesos de elaboración de dichos bloques.

- Es necesario mejorar la dosificación de materiales y procesos de elaboración de los BHC para obtener, al menos, bloques que clasifiquen como tipo NP ( $\left.\mathrm{f}^{\prime} \mathrm{b}>20 \mathrm{~kg} / \mathrm{cm}^{2}\right)$ y puedan ser usados en la tabiquería.

- $\quad \mathrm{Al}$ añadir Fpp a la mezcla se incrementa, de manera notable, la resistencia a la compresión de los BHC. Con la adición de fibras, estos BHC ya pueden ser clasificados como bloques tipo $\mathrm{P}\left(\mathrm{f}^{\prime} \mathrm{b}>50 \mathrm{~kg} / \mathrm{cm}^{2}\right)$.

- Contar con bloques que puedan trabajar de manera estructural, puede significar un cambio en los procedimientos constructivos de edificaciones que mejoren la distribución de ambientes y reduzcan los costos de ejecución de obra.

\section{Referencias bibliográficas}

Armas Aguilar, C. H. (2016). Efectos de la adición de fibra de polipropileno en las propiedades plásticas y mecánicas del concreto hidráulico. Tesis de grado, Universidad Señor de Sipán, Académico, Trujillo. doi:USSS_4547d75e72edb07b2fa76f56867d3e84

ASTM Standar C140-11a, 2012. Sampling and Testing Concrete Masonry Units and Related Units (anual Book of ASTM standars). ASTM International, West Conshohocken, PA.

Bandodkar, L., Gaonkar, A., Gaonkar,N. \& Gauns, Y. (octubre de 2011). Pulverised PET Bottles as Partial Replacement for Sand. International Journal of Earth Sciences and Engineering, 4(6), 1009-1012. Recuperado el 29 de diciembre de 2018, de https://www.researchgate.net/publication/317663447_Pulverised_PET_bottles_as_partial_replacement_of_sand

CISMID. 2001. Fabricación de bloques de concreto con una mesa vibradora. Centro Peruano Japonés de Investigaciones Sísmicas y Mitigación de Desastres. Universidad Nacional de Ingeniería.

Gallegos, H., Casabonne, C. (2006). Albañilería Estructural. Lima, Perú: Fondo Editorial PUCP.

Norma Técnica Peruana E.070: Albañilería. 2006. Reglamento Nacional de Edificaciones.

NTP 399.604 Unidades de albañilería. 2002. Métodos de muestreo y ensayo de unidades de albañilería de concreto.

Zainab Z. Ismail; Enas A. AL-Hashmi. (11 de Noviembre de 2008). Use of waste plastic in concrete mixture as aggregate replacement. Waste Management, 28(11), 2041-2047. Recuperado el 28 de diciembre de 2018, de https://www.sciencedirect.com/journal/waste-management/vol/28/issue/11 